\title{
Endocardite provoquée par Bartonella quintana, l'agent causal de la fièvre des tranchées
}

\author{
Carl Boodman MD, Terence Wuerz MD MSc (Épid.), Philippe Lagacé-Wiens MD
}

- Citation : CMAJ 2020 December 7;192:E1723-6. doi : 10.1503/cmaj.201170-f

Voir la version anglaise de l'article ici : www.cmaj.ca/lookup/doi/10.1503/cmaj.201170

$\mathbf{U}$ $n$ homme de 48 ans s'est présenté au service des urgences pour une douleur thoracique d'origine pleurétique et un essoufflement apparus 2 jours auparavant. Ses antécédents médicaux comprenaient une infection au VIH diagnostiquée 14 ans plus tôt dans le cadre d'une consommation de drogues par voie intraveineuse. Trois mois avant son admission, il avait une charge virale indétectable et une numération des CD4 de 94 cellules $/ \mathrm{mm}^{3}$ (plage normale $500-1400$ cellules $/ \mathrm{mm}^{3}$ ) ou $0,09 \times 10^{9} / \mathrm{L}$ (plage normale $0,50-1,40 \times 10^{9} / \mathrm{L}$ ). Le patient suivait le traitement antirétroviral prescrit (darunavir, ritonavir et abacavirlamivudine) et la prophylaxie contre les infections opportunistes (valacyclovir, triméthoprime-sulfaméthoxazole et fluconazole). En outre, le patient présentait une agénésie rénale unilatérale congénitale avec une fonction rénale normale à l'admission, une exposition prénatale à l'alcool et un trouble de consommation d'alcool. Il avait cessé de nombreuses années auparavant de s'injecter des drogues. À plusieurs reprises dans les 18 mois précédents, le patient avait consulté pour des épisodes de douleur thoracique et d'infestation aux poux de corps. Au moment de son admission, le patient vivait dans un logement supervisé, mais il avait vécu dans un refuge pour sans-abri dans l'année précédente.

À l'admission, le patient avait une température de $36,7^{\circ} \mathrm{C}$, une pression sanguine de $106 / 65 \mathrm{~mm} \mathrm{Hg}$, un pouls de 83 battements par minute, une fréquence respiratoire de 60 respirations par minute et une saturation en oxygène de $100 \%$ avec une oxygénothérapie à $15 \mathrm{~L} / \mathrm{min}$. L'auscultation a révélé un souffle holosystolique et un diagnostic précoce de souffle en décrescendo. À l'examen de la peau du patient, on a trouvé des lésions hémorragiques punctiformes et des lésions de grattage provenant de morsures d'arthropodes (figure 1). Il n'y avait aucune manifestation périphérique d'endocardite.

La tomodensitométrie du thorax et de l'abdomen a révélé une embolie pulmonaire, un infarctus splénique, une splénomégalie et des anévrismes des branches abdominales de l'aorte. Une échocardiographie portable localisée a indiqué une forme grave de régurgitation mitrale et une grande végétation mitrale échogène. On a prélevé 2 échantillons de sang pour hémoculture avant l'administration empirique de pipéracilline-tazobactam et de vancomycine pour une endocardite présumée.

\section{POINTS CLÉS}

- Bartonella quintana, l'agent causal de la fièvre des tranchées, se transmet par les poux de corps (Pediculus humanus corporis).

- Bien que B. quintana soit connue pour avoir causé des maladies pendant la Première Guerre mondiale, des éclosions de fièvre des tranchées se sont produites récemment chez les personnes en situation d'itinérance dans les collectivités urbaines.

- B. quintana cause l'endocardite à hémocultures négatives, qui peut être fatale si aucun traitement antimicrobien et chirurgical n'est donné, malgré les symptômes légers pendant la bactériémie chronique. Une consultation avec un infectiologue est recommandée.

- Parce que B. quintana n'est pas détecté dans les hémocultures habituelles, il faut faire une sérologie pour établir le diagnostic et demander clairement au laboratoire d'intégrer des mesures permettant d'obtenir $B$. quintana et de réaliser des tests moléculaires.

La tachypnée initiale du patient s'est apaisée avec le traitement antidouleur, et l'oxygénothérapie d'appoint a été diminuée à $2 \mathrm{~L} / \mathrm{min}$. Toutefois, 10 heures après l'admission, la tachypnée et l'hypoxémie se sont aggravées, exigeant un apport en oxygène de $15 \mathrm{~L} /$ min pour maintenir une saturation normale. En raison de l'aggravation de la détresse respiratoire, la ventilation mécanique a été amorcée, et le patient a été transféré aux soins intensifs. Une échocardiographie transœsophagienne a révélé une régurgitation mitrale grave et une végétation de $25 \mathrm{~mm} \times 9 \mathrm{~mm}$ sur la valve mitrale ainsi qu'une régurgitation aortique grave associée à de nombreuses petites végétations.

Quatre jours après son admission, le patient a subi une chirurgie de remplacement des valves aortique et mitrale en raison de lésions graves aux valves. Les résultats de pathologie ont dévoilé au moins 4 cellules polymorphonucléaires dans les tissus des 2 valves; aucun organisme n'a été identifié par coloration de Gram, et aucune croissance de pathogènes n'a été détectée dans les cultures aérobies, anaérobies et fongiques. Quatre échantillons de sang, dont 2 qui avaient été prélevées avant l'administration d'antibiotiques, ont obtenu un résultat négatif à la 
culture après 5 jours d'incubation. Aucun organisme n'a crû malgré la centrifugation du lysat et l'incubation prolongée à 6 semaines. Des sérologies ont été effectuées pour Bartonella quintana, Bartonella henselae, Coxiella burnetii et les bactéries de l'espèce Brucella, la recommandation en présence d'une endocardite à hémocultures négatives. Les sérologies pour $B$. quintana et $B$. henselae ont donné un titre d'immunoglobuline $G$ supérieur à 1:8192 (plage normale $<1: 64)^{1}$, alors que celles pour Coxiella et Brucella étaient négatives. Les résultats de séquençage de l'ARNr $16 \mathrm{~S}$ des 2 valves ont révélé du matériel génétique de $B$. quintana avec une homologie à $100 \%$ pour 741 paires de base.

Neuf jours après l'admission, le traitement antimicrobien a été modifié pour intégrer la doxycycline et la gentamicine. En raison de la détérioration de la fonction rénale, nous avons remplacé la gentamicine par la ceftriaxone après 4 jours. La ceftriaxone et la doxycycline ont été prises pendant 6 semaines après la chirurgie. Deux semaines après le remplacement des valves, le patient a reçu son congé de l'hôpital et a terminé son traitement en consultation externe. Son état demeurait stable au rendez-vous de suivi, 3 mois après son congé.

Trois autres patients infectés à $B$. quintana ont été identifiés à Winnipeg dans une période de 6 mois, et tous avaient reçu des services d'un même refuge pour sans-abri (détails aux annexes 1 et 2, accessibles en anglais au www.cmaj.ca/lookup/doi/ 10.1503/cmaj.201170/tab-related-content). Un résumé des 4 cas est présenté dans l'encadré 1 .

\section{Discussion}

B. quintana se transmet par l'inoculation des fèces de poux de corps infectés dans des lésions cutanées ${ }^{2,3}$. Historiquement, le pathogène a été associé à la fièvre des tranchées pendant la Première Guerre mondiale, mais, depuis les années 1990, des éclosions urbaines ont été signalées chez les personnes en situation d'itinérance aux États-Unis et en France ${ }^{1,4,5}$. La séroprévalence de Bartonella peut dépasser $20 \%$ chez les personnes en situation d'itinérance qui ont un trouble grave de consommation d'alcool ou qui consomment des drogues par voie intraveineuse $e^{1,4,5}$. L'endocardite à $B$. quintana est associée à un taux de mortalité de $10 \%-12 \%$ malgré un traitement antimicrobien ciblé et une chirurgie de remplacement des valves ${ }^{3,6}$. À notre connaissance, seulement 4 autres cas de $B$. quintana ont été signalés au Canada ${ }^{7,8}$.

\section{Présentation clinique}

La fièvre des tranchées est un syndrome clinique causé par $B$. quintana, qui se manifeste par de la fièvre, des maux de tête et un malaise $e^{3,6,9}$. Des splénomégalies et des ostéalgies du tibia antérieur ont été décrites ${ }^{2}$. La fièvre des tranchées était appelée à l'origine "fièvre quintane » en raison de la nature épisodique des symptômes, qui revenaient tous les 5 jours, ce qui a inspiré l'appellation $B$. quintana ${ }^{6}$. Bien qu'une bactériémie prolongée sur de nombreux mois puisse être associée à des symptômes légers et à l'absence de fièvre, elle peut aussi mener à des maladies endovasculaires, comme l'endocardite à hémocultures négatives et les anévrismes mycotiques ${ }^{5,6,9}$.

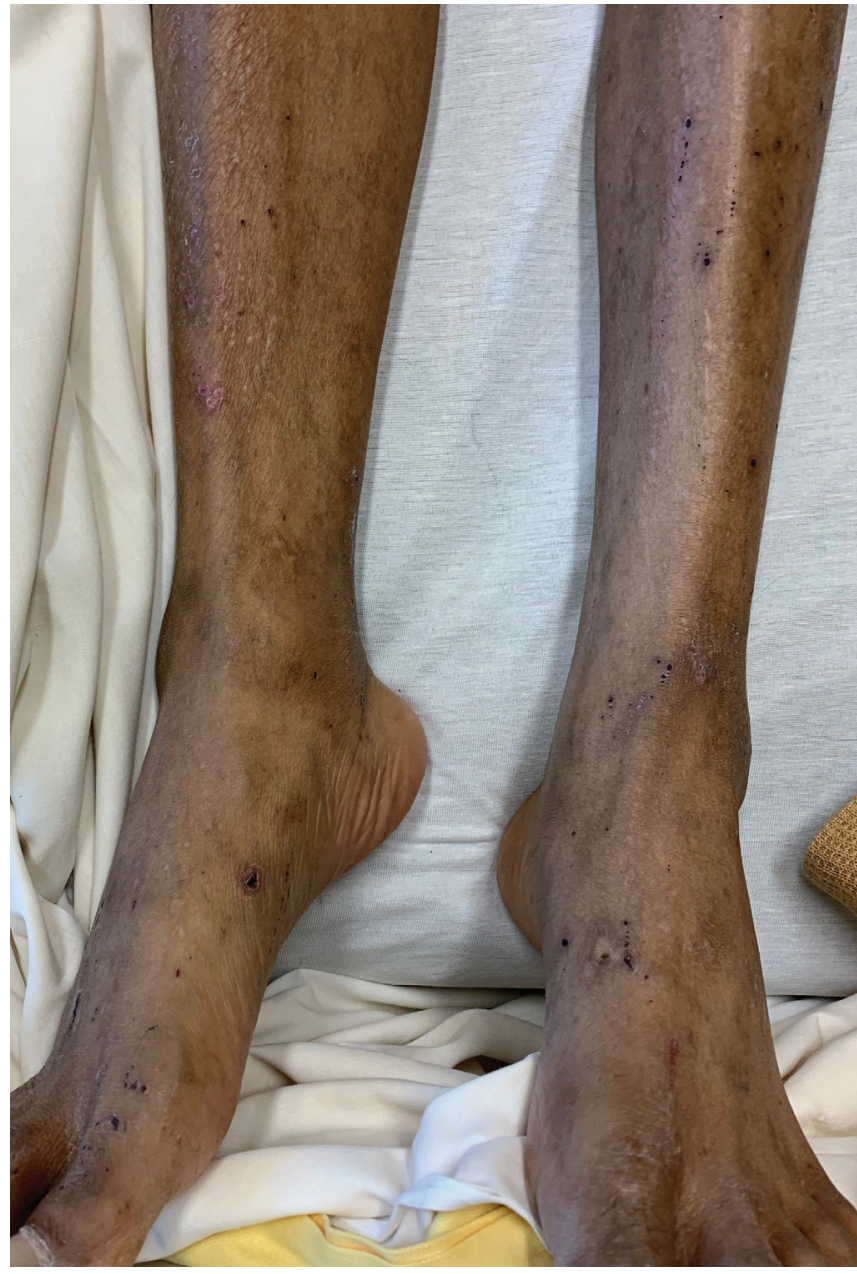

Figure 1 : Lésions hémorragiques punctiformes et lésions de grattage liées à une ectoparasitose chez un homme de 48 ans atteint d'une endocardite à Bartonella quintana (patient 1). Notons que, contrairement à celles des punaises de lit, les lésions des poux de corps ne suivent pas les points de pression, comme à la ceinture ou à l'élastique des chaussettes.

Le patient décrit ici avait consulté à plusieurs reprises pour des douleurs thoraciques et une infestation aux poux de corps, aussi appelée ectoparasitose, avant le diagnostic d'endocardite à $B$. quintana. La présentation indolente du patient, avant le développement d'une maladie embolique grave, concorde avec la bactériémie prolongée et les symptômes légers associés à B. quintana ${ }^{3,9}$.

\section{Diagnostic}

B. quintana est un bacille fastidieux à Gram négatif colonisant les érythrocytes. Une incubation prolongée allant jusqu'à 6 semaines peut être nécessaire pour isoler cet organisme dans les hémocultures $^{3,8,9}$. Comme l'incubation des hémocultures dure habituellement 5 jours dans les laboratoires de microbiologie, il est essentiel de mentionner un cas soupçonné d'infection à $B$. quintana au laboratoire afin que l'incubation soit prolongée et que des techniques supplémentaires, comme la centrifugation du lysat ou des cycles gel-dégel, soient utilisées afin de libérer les bactéries intracellulaires et d'améliorer le rendement ${ }^{8,9}$. Les tests sérologiques peuvent montrer une exposition à $B$. quintana, et un titre élevé est 
Encadré 1 : Résumé des infections à Bartonella quintana chez 4 patients à Winnipeg

\begin{tabular}{|c|c|c|c|c|}
\hline Caractéristiques & Patient 1* & Patient 2 & Patient 3 & Patient 4 \\
\hline Âge, ans & 48 & 62 & 35 & 33 \\
\hline Genre & Homme & Homme & Homme & Homme \\
\hline Syndrome diagnostiqué & $\begin{array}{l}\text { Embolisation et } \\
\text { valvulopathie }\end{array}$ & $\begin{array}{l}\text { Souffle } \\
\text { (fortuit) }\end{array}$ & $\begin{array}{l}\text { Hémorragie } \\
\text { intracrânienne (rupture } \\
\text { d'anévrisme mycotique) }\end{array}$ & $\begin{array}{l}\text { Hémorragie } \\
\text { intracrânienne (rupture } \\
\text { d'anévrisme mycotique) }\end{array}$ \\
\hline Atteinte des valves & Mitrale et aortique & Mitrale & Mitrale & Mitrale \\
\hline Antécédents de valvulopathie & Aucun & Aucun & Rhumatisme cardiaque & Aucun \\
\hline Tests diagnostiques & $\begin{array}{l}\text { Sérologie et séquençage } \\
\text { de l'ARNr } 16 \text { S sur des } \\
\text { tissus provenant des } \\
\text { valves }\end{array}$ & $\begin{array}{l}\text { Sérologie et séquençage } \\
\text { de l'ARNr } 16 \text { S sur des } \\
\text { tissus provenant des } \\
\text { valves }\end{array}$ & Sérologie & Sérologie \\
\hline Intervention chirurgicale & Remplacement valvulaire & Remplacement valvulaire & $\begin{array}{l}\text { Traitement } \\
\text { endovasculaire de l'artère } \\
\text { cérébrale }\end{array}$ & Aucune intervention \\
\hline Exposition possible & $\begin{array}{l}\text { A passé la nuit dans un } \\
\text { refuge pour sans-abri }\end{array}$ & $\begin{array}{l}\text { A passé la nuit dans un } \\
\text { refuge pour sans-abri }\end{array}$ & $\begin{array}{l}\text { A passé la nuit dans un } \\
\text { refuge pour sans-abri, } \\
\text { ectoparasitose possible } \\
\text { dans une collectivité } \\
\text { rurale }\end{array}$ & $\begin{array}{l}\text { A reçu des repas dans un } \\
\text { refuge pour sans-abri, } \\
\text { ectoparasitose possible } \\
\text { dans une collectivité } \\
\text { rurale }\end{array}$ \\
\hline $\begin{array}{l}\text { Liens avec une collectivité } \\
\text { rurale }\end{array}$ & Non & Non & Oui & Oui \\
\hline $\begin{array}{l}\text { Visite au service des urgences } \\
\text { dans les } 3 \text { mois précédant } \\
\text { l'admission à l'hôpital }\end{array}$ & Oui & Oui & Non & Oui \\
\hline Traitement antimicrobien & $\begin{array}{l}\text { - Gentamicine (4 jours) } \\
\text { - Ceftriaxone (42 jours) } \\
\text { - Doxycycline (42 jours) }\end{array}$ & $\begin{array}{l}\text { - Gentamicine (14 jours) } \\
\text { - Doxycycline (42 jours) }\end{array}$ & $\begin{array}{l}\text { - Ceftriaxone (43 jours) } \\
\text { - Vancomycine ( } 21 \text { jours) } \\
\text { - Daptomycine (22 jours) }\end{array}$ & $\begin{array}{l}\text { - Ceftriaxone (56 jours) } \\
\text { - Vancomycine (56 jours) } \\
\text { - Doxycycline (56 jours) } \\
\text { - Gentamicine (14 jours) }\end{array}$ \\
\hline Résultats & Rétablissement complet & Rétablissement complet & $\begin{array}{l}\text { Déficit cognitif léger } \\
\text { (aphasie de Broca légère) }\end{array}$ & $\begin{array}{l}\text { Déficit cognitif grave } \\
\text { (paralysie et aphasie de } \\
\text { Broca grave) }\end{array}$ \\
\hline
\end{tabular}

*Le patient 1 est le cas présenté dans le texte.

associé à l'endocardite, comme c'est le cas ici,9. Un titre dépassant 1:800 concorde avec une endocardite, et un titre plus faible concorde avec une infection chronique sans endocardite ${ }^{6}$. La sérologie est toutefois sujette à la réactivité croisée, surtout avec $B$. henselae, pathogène à l'origine de la lymphoréticulose bénigne d'inoculation, et la bactériémie chronique sans endocardite peut être associée à un titre faible ${ }^{5,9}$. Lorsqu'une sérologie est demandée pour B. quintana au Canada, des échantillons de sérum sont envoyés au Laboratoire national de microbiologie, à Winnipeg, et il faut environ 1 mois avant d'obtenir les résultats.

Les tests moléculaires de séquençage de l'ARNr $16 \mathrm{~S}$ sur les tissus des valves permettent d'établir un diagnostic d'endocardite à B. quintana. Bien que les nombreuses ressemblances génétiques entre les différentes espèces de Bartonella puissent compliquer leur identification, les tissus obtenus ici montraient une homologie à $100 \%$ avec $B$. quintana, et il y avait une séparation substantielle avec les autres espèces, y compris $B$. henselae. Une différence de plus de $0,8 \%$ avec l'espèce ayant la plus grande homologie par séquençage de l'ARNr $16 \mathrm{~S}$ suffit à faire la distinction entre les espèces. Dans le cas présent, la deuxième espèce ayant la plus grande homologie (99,03\%) était Bartonella senegalensis, qui n'est pas un pathogène humain.

L'infection à $B$. quintana peut être diagnostiquée sans confirmation de l'espèce par test moléculaire lorsqu'aucun tissu des valves n'est recueilli. Dans ce cas, il est important d'établir l'exposition potentielle aux poux de corps et d'exclure l'exposition potentielle aux chats, porteurs de $B$. henselae, qui peut causer une réactivité croisée avec $B$. quintana. Vu la rareté des demandes d'analyses de $B$. quintana, ce type d'infections est probablement sous diagnostiqué.

\section{Traitement et prévention}

Le traitement normal d'une bactériémie et d'une endocardite à B. quintana comprend une combinaison de gentamicine et de doxycycline $^{6}$. La gentamicine est habituellement administrée pendant 14 jours, en combinaison avec la doxycycline. La doxycycline 
est prise pendant 4 semaines supplémentaires en l'absence d'endocardite, et pendant au moins 6 semaines en présence d'endocardite 6 . L'inclusion de la gentamicine est associée à de meilleurs résultats cliniques et à l'élimination de la bactériémie ${ }^{6}$. Toutefois, en raison du risque lié à la toxicité de la gentamicine, des cas ont été traités avec succès par un ensemble de ceftriaxone et de doxycycline ou de rifampine $e^{6-8,10}$. Dans le cas présent, le patient a été traité par ceftriaxone sans administration prolongée d'aminoglucosides, ce qui a donné de bons résultats cliniques. D'autres études seront nécessaires pour appuyer l'utilisation de ceftriaxone et de doxycycline sans l'ajout d'aminoglucosides. Nous suggérons fortement une consultation avec un infectiologue. Comme l'indique le cas de notre patient, la triméthoprimesulfaméthoxazole ne permet pas de prévenir ou de traiter les infections à B. quintana. Des services accessibles permettant de se débarrasser des poux de corps et des autres infestations d'ectoparasites devraient être envisagés pour prévenir les infections à $B$. quintana dans les populations à risque.

\section{Conclusion}

B. quintana, transmis par les poux de corps, cause la fièvre des tranchées et peut mener à une endocardite à hémocultures négatives chez les personnes en situation d'itinérance. Les professionnels de la santé devraient envisager une sérologie pour Bartonella, une échocardiographie et une consultation avec un infectiologue lorsqu'ils prennent soin de personnes malades qui ont des antécédents d'infestations aux poux de corps. L'infection à B. quintana demeure probablement sous-diagnostiquée. La séroprévalence, la surveillance de la bactériémie et des études sur les ectoparasites sont nécessaires pour comprendre la prévalence réelle de cette infection chez les personnes en situation d'itinérance.

\section{Références}

1. Jackson LA, Spaeh DH, Kippen DA, et al. Seroprevalence to Bartonella quintana among patients at a community clinic in downtown Seattle. J Infect Dis 1996;173:1023-6.

2. OhI ME, Spach DH. Bartonella quintana and urban trench fever. Clin Infect Dis 2000;31:131-5.

3. Raoult D, Foucault C, Brouqui P. Infections in the homeless. Lancet Infect Dis 2001;1:77-84.

4. Cormer JA, Flynn C, Regnery RL, et al. Antibodies to Bartonella species in inner-city intravenous drug users in Baltimore, Md. Arch Intern Med 1996; 156:2491-5.

5. Brouqui P, Houpikian P, Dupont HT, et al. Survey of the seroprevalence of Bartonella quintana in homeless people. Clin Infect Dis 1996;23:756-9.

6. Foucault C, Brouqui P, Raoult D. Bartonella quintana characteristics and clinical management. Emerg Infect Dis J 2006;12:217-23.

7. Lam JC, Fonseca K, Pabbaraju K, et al. Bartonella quintana endocarditis outside of the Europe-African gradient: comprehensive review of cases within North America. Am J Trop Med Hyg 2019;100:1125-9.

8. Keynan Y, Mackenzie L, Lagacé-Wiens P. Quintessential culture-negative endocarditis. Can J Cardiol 2016;32:395.e9-10.

9. Brouqui $\mathrm{P}$, Lascola $\mathrm{B}$, Roux V, et al. Chronic Bartonella quintana bacteremia in homeless patients. N Engl J Med 1999;340:184-9.

10. Raoult D, Fournier P-E, Vandenesch F, et al. Outcome and treatment of Bartonella endocarditis. Arch Intern Med 2003;163:226-30.
Intérêts concurrents : Aucun déclaré.

Cet article a été révisé par des pairs.

Les auteurs ont obtenu le consentement du patient.

Affiliations : Section des maladies infectieuses (Boodman, Wuerz), Département de médecine interne et Département de microbiologie médicale et des maladies infectieuses (Boodman, Lagacé-Wiens), et Département de sciences de la santé communautaire (Wuerz), Collège de médecine Max Rady, Université du Manitoba; Soins communs Manitoba (Lagacé-Wiens), Winnipeg (Manitoba).

Contributeurs : Carl Boodman a contribué à la conception de l'article, a rédigé le manuscrit et a fourni les renseignements du cas clinique. Philippe Lagacé-Wiens a fourni un appui microbiologique à ce cas. Terence Wuerz a fourni des conseils cliniques et une supervision du cas. Tous les auteurs ont révisé de manière critique le contenu important avant de soumettre l'article. Tous les auteurs ont donné leur approbation finale pour la version soumise pour publication et assument l'entière responsabilité de tous les aspects du travail.

Propriété intellectuelle du contenu : Il s'agit d'un article en libre accès distribué conformément aux modalités de la licence Creative Commons Attribution (CC BY-NC-ND 4.0), qui permet l'utilisation, la diffusion et la reproduction dans tout médium à la condition que la publication originale soit adéquatement citée, que l'utilisation se fasse à des fins non commerciales (c.-à-d., recherche ou éducation) et qu'aucune modification ni adaptation n'y soit apportée. Voir : https://creativecommons. org/licenses/by-nc-nd/4.0/deed.fr.

Remerciements : Les auteurs remercient leurs patients pour leur attitude positive et leur volonté de participer à ce projet, et soulignent leur résilience face à des circonstances difficiles.

Correspondance : Carl Boodman, boodmanc@myumanitoba.ca; Philippe Lagacé-Wiens, plagacewiens@sharedhealthmb.ca 